\title{
Energy Conversion During Expansion of a Solid-Particle-Entrained Gas
}

\author{
C. A. Ordonez ${ }^{a}$, D. L. Weathers ${ }^{a}$, M. J. Traum ${ }^{b}$ \\ ${ }^{a}$ Department of Physics, University of North Texas, Denton, Texas 76203, USA \\ ${ }^{b}$ Department of Mechanical and Energy Engineering, University of North Texas, Denton, \\ Texas 76203, USA
}

\begin{abstract}
Pneumatic energy is converted into solid particle kinetic energy as a solidparticle-entrained gas flows downward through a vertical nozzle. For the conditions studied, the solid particles serve as a heat exchange medium to enable a gas expansion that is nearly isothermal. Also, the kinetic energy associated with the two-phase fluid is predominantly that associated with subsonic solid-particle motion. The kinetic energy of the solid particles is converted into other forms using a low-speed turbine. A theoretical description of the process is presented, and the initiation of an experimental effort to study the process is described. Experimental gas phase temperature measurements indicate the realization of isothermal expansion. Experimental turbine erosion rate measurements indicate that relatively low erosion rates occur for the conditions studied.
\end{abstract}

Keywords: gas-solid vertical flow, pneumatic energy conversion, isothermal expansion, turbine erosion 


\section{Introduction}

Gas-solid two-phase flows have been studied extensively, particularly with respect to pneumatic conveying [Klinzing et al. 2010, Sæther et al. 2009, Wypych 2008], erosion in pipes, power turbines and impeller fans [Parslow et al. 1999, Grant and Tabakoff 1975, Graham et al. 2010], and material removal processes including micro-abrasive air jet machining, sandblast finishing and air-abrasion dentistry [Li et al. 2009, Wang and Li 2003, Hegde and Khatavkar 2010]. However, solids suspended in flows moving through rotating power machinery are typically viewed as detrimental. When particles impact turbine blades, they erode the aerodynamic surfaces, leading to efficiency degradation [Hamed et al. 2006]. Unwanted solid particulates can exist within flows through turbomachinery as a consequence of combusting coal and solid biomass fuels [Wright 1987, Wright et al. 2000]. Moreover, air-breathing gas turbines can ingest particulates from the environment [Hamed et al. 2006]. The presence of particulates in gas flows through turbomachinery has motivated research on mitigating turbine blade erosion, including using protective coatings and particle separation methods [Tabakoff 1989, Musgrove et al. 2009].

A critical and novel aspect of the research reported here is that a nondetrimental view is adopted of particulates suspended in gas flows through power turbines. Solid particles are intentionally placed in the flow as a means of executing an isothermal gas expansion to maximize energy extraction while converting the energy of gas expansion to kinetic energy of the suspended particles for mechanical extraction by the turbine. Turbine erosion is mitigated by operating at a low relative speed between the suspended particles and the turbine surfaces.

Past evaluations of solid/gas flows through turbomachinery provide guid- 
ance for the current effort. Ghenaiet et al. investigated the erosion of axial fans from particulate ingestion using a single-stage contra-whirl axial fan made from cast aluminum and driven by an electric motor [Ghenaiet et al. 2004]. The fan was painted, and erosion locations were experimentally identified by sites of paint removal. In addition, fan material erosion as a function of time and as a function of particulate mass injected in the gas flow were measured.

To measure the pressure drop in vertical gas-solid two-phase mixtures within monolith channels, Ding et al. created a particle injection unit and demonstrated measurement techniques that prove instructive for the current apparatus [Ding et al. 2005]. Static gas pressure was measured using pressure taps, and pressure drop across the monolith was measured with a micro-manometer. Particle flow rate was measured by weighing particles collected from the experiment over time.

Guidance on particle size selection arises from the gravity-fed silo sand discharge experiments of Hsiau et al. [Hsiau et al. 2010]. In a closed silo configuration, solid and gas phases flow in opposite directions; the particles fall under gravity and air moves at subsonic velocity past them to fill the void. While closed silo counter flow differs from the co-flow of the current system, the silo example suggests particle radius selection of order $100 \mu \mathrm{m}$. In solid/gas mixtures under conditions similar to the current system, particles smaller than a few hundred $\mu \mathrm{m}$ in radius tend to present larger flow resistance to air than larger particles. In the co-flow configuration of the current system, such an effect would tend to increase particle acceleration up to gas velocity, owing to low air permeability through the suspension. Be that as it may, it must also be considered that employing particles smaller than $100 \mu \mathrm{m}$ may have a tendency to foul a particle/gas separating filter. 
The two-phase flow direction is oriented vertically, parallel to the gravity vector. This orientation avoids the possibility of sedimentation of the solid phase under gravity [Yan and Koplik 2009]. Guidance on theory development is provided by prior theoretical research on pneumatic conveyance [Klinzing et al. 2010], and, in particular, abrasive air jets [Li et al. 2009], although an adiabatic gas expansion treatment is typically used for abrasive air jets.

The system studied in the present work is illustrated conceptually in Fig. 1. A set of parameter values is given by way of example in Table 1. The energy conversion process can be divided into parts. In the first part of the process, a fluid comprising a mixture of gas and solid particles accelerates down a nozzle. The acceleration part of the process is describable as the expansion of a two-phase fluid that flows downward through a vertical nozzle.

Some important features of the first part of the process include the following: (1) The gas and solid particles accelerate down the nozzle at approximately equal rates. (2) The post-acceleration kinetic energy associated with the two-phase fluid motion is predominantly that associated with subsonic solid-particle motion. (3) The temperatures of the gas and solid particles remain approximately equal during an isothermal expansion of the gas.

In the second part of the process, the two-phase fluid is directed at a turbine, which is connected to a dynamometer. Because the solid particles are essentially incompressible and will be traveling at a speed much slower than the speed of sound, a low-speed turbine for use with incompressible fluids is suitable. Such turbines can, in principal, achieve a high energy conversion efficiency in the limit that all of the solid particles have the same velocity. 
In the third part of the process, the solid particles are separated from the expanded gas and recycled. Energy must be expended to transport the particles and to pressurize or re-pressurize the system. If the solid particles are large enough, which is the case for the parameters listed in Table 1, gravity and filtering can be used to separate the solid particles from the expanded gas.

Pneumatic energy conversion processes often occur at much lower efficiency than hydraulic and electric energy conversion processes. It is anticipated that a solid-particle-entrained gas may eventually serve for converting pneumatic energy into other forms with high efficiency.

\section{Discrete Characteristics of the Flow}

A cylindrical coordinate system is defined within a nozzle shaped as a cylindrical tube, with the $z$ axis coincident with the axis of symmetry of the nozzle. The description is developed in one dimension. Physical quantities may have a dependence on the axial coordinate $z$, but any dependence that quantities may have on the radial and azimuthal cylindrical coordinates is neglected. For brevity, physical quantities that have a dependence on the axial coordinate $z$ are not explicitly shown as having such a dependence. The solid particles are assumed to be spherical and to have a radius $r_{s}$ that is much larger than the effective radius $r_{g}$ of a gas molecule. With $r_{s} \gg r_{g}$, a gas molecule has a mean free path between collisions with solid particles given by $\lambda_{g s}=1 /\left(n_{s} \pi r_{s}^{2}\right)$ if the gas is not present, where $n_{s}$ is the number of solid particles per unit volume. A solid particle has a mean free path between collisions with other solid particles given by $\lambda_{s}=1 /\left[n_{s} \pi\left(2 r_{s}\right)^{2}\right]=\lambda_{g s} / 4$ if the gas is not present. A gas molecule has a mean free path between collisions 
with other gas molecules given by $\lambda_{g}=1 /\left[n_{g} \pi\left(2 r_{g}\right)^{2}\right]=1 /\left(n_{g} 4 \pi r_{g}^{2}\right)$ if the solid particles are not present, where $n_{g}$ is the number of the gas molecules per unit volume.

Consider an axial length $\delta L$ of the two-phase fluid that is small enough for the properties of the fluid to be approximated as uniform anywhere within an associated cylindrical volume $V$, but that is much larger than the typical distances $n_{g}^{-1 / 3}$ and $n_{s}^{-1 / 3}$ between nearest-neighbor gas molecules and solid particles, respectively. Let $N_{g}=n_{g} V$ denote the number of gas molecules within the volume $V$. The volume $V$ is defined such that $N_{g}$ is constant. Let $N_{s}=n_{s} V$ denote the number of solid particles within $V$. The value of $N_{s}$ is also approximately constant under the conditions considered.

The volume $V$ is divided into two parts: $V=V_{g}+V_{s}$. Here, $V_{g}$ refers to the part of the volume in which the gas molecules can move (i.e., the volume outside the solid particles) and $V_{s}$ refers to the volume occupied by the solid material. The values of $V_{g}$ and $V$ change as a function of $z$, and the value of $V_{s}$ is treated as being constant. Define a parameter $R_{V}=V_{s} / V_{g}$, which represents the ratio of the volume occupied by the solid particles and the volume that contains the gas molecules. The number density of solid particles is $n_{s}=N_{s} / V=N_{s} /\left(V_{g}+V_{s}\right)=N_{s} /\left[V_{s}\left(1+1 / R_{V}\right)\right]$. Identifying $V_{s} / N_{s}=(4 / 3) \pi r_{s}^{3}$ as the volume of one solid particle gives

$$
n_{s}=\frac{3}{4 \pi r_{s}^{3}\left(1+1 / R_{V}\right)}
$$

and

$$
\lambda_{g s}=\frac{4}{3} r_{s}\left(1+\frac{1}{R_{V}}\right) .
$$

There is more than one way to define the number density of one phase of a two-phase fluid. For example, the number density of gas molecules within 
the fluid considered here can be defined as either $N_{g} / V$ or $N_{g} / V_{g}$. The first definition is used within the present work. However, the second definition must be used within the ideal gas law, $p V_{g}=N_{g} k T$, which is assumed to apply to the gas phase. Here, $p$ and $T$ are the pressure and temperature associated with the gas phase at coordinate $z$, and $k$ is Boltzmann's constant. Thus, with $n_{g}=N_{g} / V=N_{g} /\left(V_{g}+V_{s}\right)=N_{g} /\left[V_{g}\left(1+R_{V}\right)\right]$, substitution using the ideal gas law yields

$$
n_{g}=\frac{p}{k T\left(R_{V}+1\right)}
$$

and

$$
\lambda_{g}=\frac{k T\left(R_{V}+1\right)}{4 \pi r_{g}^{2} p} .
$$

Suppose the gas phase of the fluid expands isothermally at temperature $T$ as the fluid travels down the nozzle. The conditions required for such an expansion are evaluated below. At the beginning of the expansion process, $R_{V i}=V_{s} / V_{g i}$, where subscript $i$ indicates initial value. At the end of the expansion process, $R_{V f}=V_{s} / V_{g f}$, where subscript $f$ indicates final value. For isothermal expansion of gas, the ideal gas law gives $p_{f} V_{g f}=p_{i} V_{g i}$. Thus, $R_{V f}=\left(p_{f} / p_{i}\right) V_{s} / V_{g i}=\left(p_{f} / p_{i}\right) R_{V i}$. By way of example, the following parameter values are considered: $r_{g}=0.16 \mathrm{~nm}$ (e.g., nitrogen), $r_{s}=100 \mu \mathrm{m}$, $T=298 \mathrm{~K}, p_{i}=0.79 \mathrm{MPa}, p_{f}=0.10 \mathrm{MPa}, R_{V i}=1.35$ (measured), and $R_{V f}=\left(p_{f} / p_{i}\right) R_{V i}=0.17$. The mean free paths have the values, $\lambda_{g}=38$ $\mathrm{nm}, \lambda_{s}=58 \mu \mathrm{m}$, and $\lambda_{g s}=230 \mu \mathrm{m}$, at the start of the expansion, and $\lambda_{g}=150 \mathrm{~nm}, \lambda_{s}=230 \mu \mathrm{m}$, and $\lambda_{g s}=920 \mu \mathrm{m}$, at the end of the expansion. The following relationships occur for the parameter values considered: $\lambda_{g} \ll \lambda_{s}<\lambda_{g s} \ll r_{n} \ll L_{n}$. Here, $r_{n}$ and $L_{n}$ are the inner radius and length of the nozzle. 


\section{Continuous Characteristics of the Flow}

With the definitions used for the number density of each phase of the two-phase fluid, $n_{g}=N_{g} / V$ and $n_{s}=N_{s} / V$, a (total) number density can be conveniently expressed as $n=n_{g}+n_{s}$. Similarly, the mass density of the two-phase fluid is expressed as $\rho=\rho_{g}+\rho_{s}$, with the mass densities of the gas and solid phases defined as $\rho_{g}=m_{g} N_{g} / V=m_{g} n_{g}$ and $\rho_{s}=m_{s} N_{s} / V=$ $m_{s} n_{s}$. Here $m_{g}$ and $m_{s}$ are the masses of a gas molecule and solid particle, respectively. Likewise, the drift kinetic energy density of the two-phase fluid is expressed as $\kappa=\kappa_{g}+\kappa_{s}$, with $\kappa_{g}=\frac{1}{2} \rho_{g} v_{g}^{2}$ and $\kappa_{s}=\frac{1}{2} \rho_{s} v_{s}^{2}$, where $v_{g}$ and $v_{s}$ are the average axial speeds of the gas molecules and solid particles, respectively.

The ratio of the drift kinetic energy of the fluid associated with the solid phase to that associated with the gas phase is now evaluated, assuming that the conditions within the nozzle are such that the solid particles can be treated as having an average axial speed that equals the gas flow speed, $v_{s}=$ $v_{g}$. The assumption is checked below. The ratio is $R_{\kappa}=\kappa_{s} / \kappa_{g}=\rho_{s} / \rho_{g}=$ $m_{s} n_{s} /\left(m_{g} n_{g}\right)=3 m_{s} k T R_{V} /\left(4 \pi r_{s}^{3} m_{g} p\right)$. To proceed further, it is convenient to distinguish between $\rho_{s}$ and a material mass density $\rho_{m s}$ associated with the material that composes the solid particles. The latter is related to the mass of a solid particle by $m_{s}=\frac{4}{3} \pi r_{s}^{3} \rho_{m s}$. In terms of $\rho_{m s}$, the ratio is

$$
R_{\kappa}=\frac{\rho_{m s} k T R_{V}}{m_{g} p} .
$$

By way of example, the following parameter values from Table 1 are considered: $m_{g}=28 \mathrm{u}$ (nitrogen), $\rho_{m s}=8000 \mathrm{~kg} / \mathrm{m}^{3}$ (stainless steel), $T=298 \mathrm{~K}$, $p_{i}=0.79 \mathrm{MPa}, p_{f}=0.10 \mathrm{MPa}, R_{V i}=1.35$, and $R_{V f}=0.17$. The value $R_{\kappa}=1200$ is obtained at both the start and end of the expansion. The same 
value occurs because $R_{V i} / p_{i}=R_{V f} / p_{f}$ for an isothermal expansion of the gas phase.

The work done by the gas phase of the fluid during an isothermal increase in volume from $V_{g i}$ to $V_{g f}$ is $W_{f}=\int_{V_{g i}}^{V_{g f}} p d V_{g}=N_{g} k T \int_{V_{g i}}^{V_{g f}} V_{g}^{-1} d V_{g}=$ $N_{g} k T \ln \left(V_{g f} / V_{g i}\right)=N_{g} k T \ln \left(p_{i} / p_{f}\right)$. The isothermal gaseous specific work, defined as the work per unit mass of gas, is $\omega_{g f}=W_{f} /\left(m_{g} N_{g}\right)=\left(k T / m_{g}\right) \ln \left(p_{i} / p_{f}\right)$. The isothermal work density is defined as $w_{f}=W_{f} / V_{f}=n_{g f} k T \ln \left(p_{i} / p_{f}\right)$. The work done by the gas phase increases the fluid's drift kinetic energy. The fluid's drift kinetic energy density after expansion is $\kappa_{f}=\eta w_{f}$, provided that the initial drift kinetic energy density of the fluid is negligible, $\kappa_{i} \ll \kappa_{f}$, and provided further that the effect of gravity is negligible. Here, $\eta$ accounts for losses such as dissipative losses, and it has a value less than unity, $\eta<1$. A value for $\eta$ is to be assessed experimentally in future work by comparison with theoretical predictions presented here using $\eta=1$. For the parameters considered in Table 1, the fluid's drift kinetic energy is predominantly that associated with the solid phase so that $\kappa_{f}=\kappa_{s f}$. With $\kappa_{s f}=\frac{1}{2} m_{s} n_{s f} v_{s f}^{2}$ and $R_{\kappa}=m_{s} n_{s} /\left(m_{g} n_{g}\right)$, the maximum speed of the solid particles (at the end of expansion) is found to be

$$
v_{s f}=\sqrt{\frac{2 k T \eta \ln \left(p_{i} / p_{f}\right)}{m_{g} R_{\kappa}}},
$$

which has a value of $17 \mathrm{~m} / \mathrm{s}$ for the values considered in Table 1 . The fluid flow is subsonic for the values considered. The fluid's maximum kinetic power (at the end of expansion) is

$$
P_{f}=\kappa_{s f} \pi r_{n}^{2} v_{s f}
$$

which has a value of $240 \mathrm{~W}$ for the values considered in Table 1 . 
Let $\Delta v=v_{g}-v_{s}$ denote the slip speed. The speed quotient $f_{\Delta v}=\Delta v / v_{s}$ serves to characterize the proximity to equal flow speeds for the two phases. Assuming that the ideal gas law applies to the gas phase, the drag force on a single solid spherical particle is

$$
F_{d}=\frac{C_{d} m_{g} p \pi r_{s}^{2}(\Delta v)^{2}}{2 k T}
$$

where $C_{d}$ is the coefficient of drag. To solve for $\Delta v$, work associated with gas expansion is assumed to go predominantly into increasing the kinetic energy of the solid phase. Assuming isothermal expansion, the work done by the gas phase of the fluid during a decrease in pressure from $p_{i}$ to $p$ is $W=N_{g} k T \ln \left(p_{i} / p\right)$. A constant pressure gradient within the nozzle is assumed,

$$
\frac{d p}{d z}=\frac{p_{f}-p_{i}}{L_{n}}
$$

The net force in the $z$ direction that is imparted on the two-phase fluid as a result of gas expansion is

$$
F=\frac{d W}{d z}=\frac{\partial W}{\partial p} \frac{d p}{d z}=\frac{N_{g} k T}{L_{n}}\left(\frac{p_{i}-p_{f}}{p}\right) .
$$

The net force in the $z$ direction per solid particle is

$$
F_{s}=\frac{F}{N_{s}}=\frac{n_{g} k T}{n_{s} L_{n}}\left(\frac{p_{i}-p_{f}}{p}\right)=\frac{m_{s} k T}{m_{g} R_{\kappa} L_{n}}\left(\frac{p_{i}-p_{f}}{p}\right)=\frac{4 \pi r_{s}^{3} \rho_{m s} k T}{3 m_{g} R_{\kappa} L_{n}}\left(\frac{p_{i}-p_{f}}{p}\right) .
$$

Requiring $F_{d}=F_{s}$ and solving for $\Delta v$ gives

$$
\Delta v=\frac{k T}{m_{g} p} \sqrt{\frac{8 \rho_{m s} r_{s}\left(p_{i}-p_{f}\right)}{3 C_{d} L_{n} R_{\kappa}}} .
$$

Evaluation of the speed quotient $f_{\Delta v}=\Delta v / v_{s}$ gives an expression that increases as the expansion progresses after the pressure has dropped by at least 
$39 \%$. The value at the end of the expansion is given by

$$
f_{\Delta v}=\sqrt{\frac{4 \rho_{m s} r_{s}\left(p_{i}-p_{f}\right) k T}{3 m_{g} C_{d} L_{n} \eta p_{f}^{2} \ln \left(p_{i} / p_{f}\right)}} .
$$

By way of example, the parameter values in Table 1 are considered. The coefficient of drag is approximated as having a value of 0.5 , which should be suitable for the applicable range of Reynolds number values. The value

obtained is $f_{\Delta v}=4.6$, which indicates that Eq. (5) overestimates the value $R_{\kappa}=1200$ by a factor of $\left(v_{g} / v_{s}\right)^{2}=\left(f_{\Delta v}+1\right)^{2}=31$ at the end of the expansion. Thus, the value of $R_{\kappa}$ is nevertheless much larger than unity. It is concluded that, for the parameter values listed in Table 1, the drift kinetic energy associated with the fluid motion is predominantly that associated with the solid phase.

\section{Thermal Characteristics of the Flow}

The heat capacity of a volume $V$ of the two-phase fluid is divided into parts associated with the gas and solid phases: $C_{g}=m_{g} N_{g} c_{g}$ and $C_{s}=$ $m_{s} N_{s} c_{s}$. Here, $c_{g}$ and $c_{s}$ are the specific heat capacities of the gas and solid phases of the fluid, respectively. The ratio of the heat capacities associated with the two fluid phases is $R_{C}=C_{s} / C_{g}=m_{s} n_{s} c_{s} /\left(m_{g} n_{g} c_{g}\right)$ or

$$
R_{C}=\frac{c_{s} R_{\kappa}}{c_{g}} .
$$

By way of example, the parameter values in Table 1 are considered: $R_{\kappa}=$ $1200, c_{s}=500 \mathrm{~J} / \mathrm{kg} \cdot \mathrm{K}$, and $c_{g}=743 \mathrm{~J} / \mathrm{kg} \cdot \mathrm{K}$. The value obtained is $R_{C}=810$. The value used for $c_{s}$ is the specific heat capacity for 304 stainless steel at $T=298 \mathrm{~K}$ and atmospheric pressure. An isothermal gas expansion process can be approximated as a succession of adiabatic expansions and constantvolume temperature increases. The value used for $c_{g}$ is the specific heat 
capacity at constant volume for nitrogen at $T=298 \mathrm{~K}$ and atmospheric pressure. The values for $c_{s}$ and $c_{g}$ may be expected to be approximately constant for the range of pressures considered in Table 1. A value of $R_{C}$ much larger than unity indicates that the heat capacity associated with the solid phase of the fluid is much larger than that associated with the gas phase.

For an isothermal expansion to occur, heat must flow from the solid phase of the fluid to the gas phase. The amount of heat energy $H$ that must flow equals the difference between the thermal energy of the gas phase if an isothermal expansion occurs and the thermal energy of the gas phase if an adiabatic expansion occurs. The thermal energy of the gas phase in a volume $V$ of the fluid before expansion takes place is $H_{i}=\frac{3}{2} N_{g} k T_{i}$. If the expansion is isothermal, then the thermal energy of the gas phase in a volume $V$ of the fluid after expansion takes place is also $H_{i}$. If the expansion is adiabatic, then $p V^{\gamma}=p_{i} V_{i}^{\gamma}$, and the thermal energy of the gas in a volume $V$ of the fluid after an arbitrary amount of expansion takes place is $H_{a}=$ $\frac{3}{2} N_{g} k T_{i}\left(p / p_{i}\right)^{(1-1 / \gamma)}$. Here, $\gamma$ is the ratio of the gas' heat capacities at constant pressure and constant volume. ( $\gamma=1.4$ for an ideal diatomic gas.) The amount of heat energy $H$ that must flow from the solid phase to the gas phase for an isothermal $\left(T=T_{i}\right)$ expansion to occur is $H=H_{i}-H_{a}=\frac{3}{2} N_{g} k T[1-$ $\left.\left(p / p_{i}\right)^{(1-1 / \gamma)}\right]$. The associated rate at which heat energy is transferred from the solid phase to the gas phase within a volume $V$ of the fluid is

$$
\dot{H}=\frac{d H}{d t}=\frac{\partial H}{\partial p} \frac{\partial p}{\partial z} \frac{d z}{d t} .
$$

The first term on the right is evaluated as

$$
\frac{\partial H}{\partial p}=\frac{-3 N_{g} k T\left(p_{i} / p\right)^{(1 / \gamma)}(1-1 / \gamma)}{2 p_{i}} .
$$


A constant pressure gradient within the nozzle is assumed, as given by Eq. (9). The approximate axial drift speed of the fluid, $\frac{d z}{d t}=v_{s}$, is obtained by equating $\eta w=\eta n_{g} k T \ln \left(p_{i} / p\right)$ and $\kappa_{s}=\frac{1}{2} m_{s} n_{s} v_{s}^{2}$. Substitution of terms gives an expression for $\dot{H}$, which increases as the expansion progresses. The largest rate, which occurs at the end of the expansion, is given by

$$
\dot{H}_{f}=\frac{3 N_{g} k T\left(p_{i} / p_{f}\right)^{(1 / \gamma)}(1-1 / \gamma)}{L_{n}}\left(1-\frac{p_{f}}{p_{i}}\right) \sqrt{\frac{\eta k T \ln \left(p_{i} / p_{f}\right)}{2 m_{g} R_{\kappa}}} .
$$

The thermal conductivity of 304 stainless steel is $K_{s}=16 \mathrm{~W} / \mathrm{m} \cdot \mathrm{K}$ and that for nitrogen gas is $K_{g}=0.025 \mathrm{~W} / \mathrm{m} \cdot \mathrm{K}$ for the parameters considered in Table 1. Because of the disparity in thermal conductivities, the temperature gradient in the gas surrounding a solid particle is assumed to be much larger than the temperature gradient within the solid particle. The temperature within a solid particle is approximated as being spatially uniform and is denoted $T_{s}$. The temperature $T_{s}$ is also approximated as being temporally constant, because the heat capacity associated with the solid phase of the fluid is much larger than that associated with the gas phase. The temperatures of both phases are taken to be equal before the expansion begins.

The temperature gradient within the gas is approximated as occurring within a distance of order $r_{s}$ from a solid particle. For larger distances, convection (e.g., associated with a finite relative drift between the solid phase and the gas phase) is assumed to dominate the heat transport. The temperature within the gas at distances larger than $\sim r_{s}$ from a solid particle is approximated as being spatially uniform and is denoted $T_{g}$. If an isothermal expansion occurs, then the difference in temperatures $\Delta T=T_{s}-T_{g}$ is much smaller than either temperature, and both temperatures are approximately equal, $T \approx T_{s} \approx T_{g}$. The fraction $f_{\Delta T}=\Delta T / T$ serves to characterize the 
proximity to isothermal expansion. The rate at which heat energy is conducted from the solid phase to the gas phase within a volume $V$ of the fluid is of order $\dot{H}_{c}=K_{g} A_{s} \Delta T / r_{s}$. Here, $A_{s}=4 \pi r_{s}^{2} N_{s}$ is the surface area of the solid particles. The rate $\dot{H}_{c}$ decreases as the expansion progresses. Consequently, if the condition $\dot{H}=\dot{H}_{c}$ for isothermal expansion is met at the end of the expansion, then the condition is met throughout the expansion. Imposing

the condition $\dot{H}=\dot{H}_{c}$ at the end of the expansion requires $f_{\Delta T f} \sim 10^{-2}$ for the parameters considered in Table 1. Such a value is sufficiently small for the expansion to be describable as isothermal.

\section{Initial Experimentation}

An experiment on the first and second parts of the energy conversion process has been initiated, using parameters similar to those given in Table 1. Figure 2 shows a photograph of the primary components of the apparatus. To operate the experiment, a discharge tank is manually loaded with solid particles and then pressurized with gas. A valve is opened to allow the two-phase fluid to enter a nozzle, through which the fluid expands. The accelerated fluid drives a turbine that is connected to a dynamometer. Steps were taken to avoid operating the apparatus with the discharge tank depleted of solid particles. (Free flowing gas could produce a much larger power and might spin up the turbine employed to the point of catastrophic failure.) Conventional diagnostics are used for measuring temperature, pressure, flow rate, turbine rotation speed, and dynamometer torque. The principal of operation was clearly demonstrated. For this initial experimentation, the energy conversion efficiency was found to be low, a few percent at most, because of the type of turbine employed. A substantial fraction of the fluid 
would pass between the blades unimpeded. A more efficient impulse turbine is to be acquired for future implementation. The dynamometer provides a measurement of the applied torque for a chosen frictional brake load. In principal, the load can be chosen so as to maximize the energy conversion efficiency. The most efficient operation occurs at a rotation speed equal to about half the free-spin speed.

Complete integration of the third part of the process is also planned for future work. For the solid particles to be recycled, they must be returned to the initial thermodynamic state in which they are mixed with compressed gas to form a two-phase fluid. One possible configuration incorporates two discharge tanks that are cyclically pressurized out of phase. Pneumatic conveying is used for refilling a discharge tank at times when the discharge tank is depressurized. Energy must be expended to transport the particles and re-pressurize each discharge tank, and the associated energy costs must be taken into account for experimentally evaluating the overall energy conversion efficiency of the integrated system.

To achieve isothermal expansion, heat must be transferred from the solid particles to the gas. In effect, the particles are cooled slightly by the interaction. For continuous operation using a high-temperature compressed gas (e.g., that is produced from combustion), thermal insulation may be incorporated effectively to put an adiabatic boundary around the entire device. Now the solid particles cool slightly during each expansion process and are reheated while in the discharge tank, which may also serve as a combustion chamber. For continuous operation using an ambient-temperature compressed gas, the solid particles may be reheated by heat exchange with an ambient-temperature thermal reservoir (e.g., air), while being transported 
back to the discharge tank.

The temperature of the gas phase after expansion through the nozzle was measured within the particle separation chamber with the turbine removed. The temperature was measured outside the flow stream using a thermocouple thermometer with a $\pm 0.5 \mathrm{~K}$ accuracy and a $1 \mathrm{~s}$ response time. The thermocouple was inserted through the turbine port, which was otherwise sealed. The particles were loaded into the apparatus' discharge tank, which was then pressurized to $0.38 \mathrm{MPa}$ with compressed air and maintained at that value during the experiment by a pressure regulator. The experiment consisted of opening the nozzle valve and recording the time-dependent temperature for a time period much longer than the thermometer response time. It was found that the temperature remained within $1 \mathrm{~K}$ of ambient temperature under nominal operating procedures. For one measurement, the apparatus was left alone for more than 24 hours within the temperature-controlled laboratory. The temperatures of all primary components including the compressor tank and the solid particles were measured before and after the gas expansion using an infrared thermometer, which indicated that everything was at room temperature. It was confirmed that the temperature measured by the thermocouple thermometer remained within $1 \mathrm{~K}$ of ambient temperature during the gas expansion. In contrast, an ideal adiabatic expansion would have caused the temperature of the expanded gas to decrease by $90 \mathrm{~K}$. The result is interpreted as indicating the realization of an isothermal expansion of the gas phase.

An erosion rate for the turbine in Fig. 2 was measured. The turbine (316 stainless steel; $7.6 \mathrm{~cm}$ diameter) was first thoroughly cleaned using a fine-hair brush and blasted with compressed air to remove adhered particle 
contaminants. It was weighed using a Mettler-Toledo XS205 precision balance set to $0.01 \mathrm{mg}$ resolution to establish a baseline mass. The turbine was then immersed in a particle-filled hopper for about five seconds; was then removed, brushed down, blown clean with compressed air, and re-weighed. This process was repeated multiple times to establish the turbine's average mass (with experimental uncertainty), $108.4978 \pm 0.0011 \mathrm{~g}$, subject to exposure to the hopper full of solid particles. As the solid particles had no velocity, this procedure should not erode the turbine. Experimental uncertainty larger than the resolution of the balance is attributed to adhesion to the turbine's surface of unobserved contaminants resulting from alternating exposure to the particle hopper, the cleaning processes, and the laboratory environment.

The turbine was fixed in place within the experimental apparatus so it could not rotate, and one blade was positioned directly under the nozzle. In normal operation, the turbine would be spinning, and its blades would present a relative velocity to the particles lower in magnitude than when the blades are fixed. Higher relative velocity between the particles and blades corresponds to more prevalent blade erosion. Therefore, by preventing turbine rotation in this test, maximum relative velocity is achieved, and the result is an upper bound on the expected erosion rate for the real functioning system. After the particles were loaded into the apparatus' discharge tank, its pressure was kept at $0.38 \mathrm{MPa}$ during the experiment. The experiment commenced by opening a valve, which trained a jet of particles on the fixed turbine blade at constant volume flow rate. After striking the fixed turbine blade, the particles were collected in the particle separation chamber and ultimately weighed using an Ohaus Scout Pro balance (with 1 g resolution) 
to establish the weight of particles used. As the experiment was not rigged for continuous operation, the process was repeated three times, streaming a total of $22,273 \pm 15$ grams of particles over the fixed turbine blade.

Between each streaming event (each event used approximately 7500 grams of particles), the turbine was recovered from the apparatus and its set screws removed to release particles that may have accumulated in the threads. To eliminate additional adhered particles, the turbine was tapped against a solid surface, cleaned with the fine-hair brush, and blasted with compressed air. It was then weighed on the Mettler-Toledo XS205 precision balance to establish mass lost due to erosion. Reported results are the cumulative outcome from all three identical particle streaming events.

The final turbine mass, $108.4958 \pm 0.0011$ grams, indicates a total mass reduction from erosion of no more than $0.0020 \pm 0.0022$ grams. The erosion mass with respect to the streamed particle mass was $9.0 \times 10^{-5} \pm 9.9 \times 10^{-5}$ $\mathrm{mg} / \mathrm{g}$. As a basis of comparison, this erosion rate is over 6600 times smaller than a rate reported on the same unit basis by Ghenaiet et al. for MILE5007E sand particles (0 - $1000 \mu \mathrm{m}$ diameter) impacting a rotating cast aluminum impeller [Ghenaiet et al. 2004, Ghenaiet et al. 2005]. The lower erosion rate is attributed primarily to a lower relative speed between the suspended particles and the blade surface, although other factors may be important as well. By way of comparison, the lifetime of a turbine in our system is approximated by extrapolating the efficiency drop Ghenaiet et al. observed over 9 hours at an average erosion rate of $0.843 \mathrm{mg} / \mathrm{g}$. This extrapolation suggests similar degradation might be expected for the turbine of our system after 9.52 years. Moreover, the $7.1 \%$ adiabatic efficiency reduction Ghenaiet et al. observed after 9 hours resulted primarily from aerodynamic 
performance degradation. The aerodynamic characteristics of our subsonic turbine are not critical to its proper operation, and performance degradation of our turbine should therefore be slower than the aerodynamic degradation observed by Ghenaiet et al. Recall also that our measurement yields the maximum erosion rate because the turbine was fixed. For these reasons, the true turbine performance degradation for our system should be much slower than the $0.75 \%$ /year reduction (at $100 \%$ utilization) suggested by the analysis. It is concluded that manageable erosion rates may be possible for the system illustrated in Fig. 1.

\section{Conclusion}

Discrete, continuous and thermal characteristics of a gas-solid downward flow through a vertical nozzle have been developed under low-slip, isothermal expansion conditions. Number densities, which provide typical distances between nearest-neighbor solid particles or gas molecules, and mean free paths were evaluated to characterize some of the scale lengths involved. The drift kinetic energy and heat capacity were found to be predominantly associated with the solid phase under the conditions studied. Expressions for the maximum (ideal) speed and kinetic power of the solid phase at the end of expansion were derived, and provide a predictive benchmark for experimentally determined values. Methods were developed to check that the conditions for

low-slip and isothermal expansion were satisfied. Experimentation was initiated, and energy conversion of a gas-solid flow was clearly demonstrated using a turbine. The gas-phase temperature was measured after expansion, and the result was consistent with isothermal expansion. An erosion rate for the turbine was measured, and the result was consistent with a manageable 
erosion rate.

\section{Acknowledgments}

The authors would like to thank E. Lessmann for help with the experimental effort. 


\section{References}

[Ding et al. 2005] Ding, Y., Z. Wang, M. Ghadiri, and D. Wen. 2005. Vertical Upward Flow of Gas-Solid Two-Phase Mixtures Through Monolith Channels. Powder Technology 153, 51-58.

[Ghenaiet et al. 2004] Ghenaiet, A., S. C. Tan, and R. L. Elder. 2004. Experimental Investigation of Axial Fan Erosion and Performance Degradation. Proceedings of the Institution of Mechanical Engineers, Part A: Journal of Power and Energy, 218, 437-450.

[Ghenaiet et al. 2005] Ghenaiet, A., S. C. Tan, and R. L. Elder. 2005. Prediction of an Axial Turbomachine Performance Degradation Due to Sand Ingestion. Proceedings of the Institution of Mechanical Engineers, Part A: Journal of Power and Energy, 219, 273-287.

[Graham et al. 2010] Graham, L. J. W., D. R. Lester, and J. Wu. 2010. Quantification of Erosion Distributions in Complex Geometries. Wear 268, 1066-1071.

[Grant and Tabakoff 1975] Grant, G., and W. Tabakoff. 1975. Erosion Prediction in Turbomachinery Resulting from Environmental Solid Particles. Journal of Aircraft 12, 471-478.

[Hamed et al. 2006] Hamed, A., W. Tabakoff, and R. Wenglarz. 2006. Erosion and Deposition in Turbomachinery. Journal of Propulsion and Power 22, 350-360. 
[Hegde and Khatavkar 2010] Hegde, V.S., and R. A Khatavkar. 2010. A New Dimension to Conservative Dentistry: Air Abrasion. Journal of Conservative Dentistry $13,4-8$.

[Hsiau et al. 2010] Hsiau, S., C. Hsu, and J. Smid. 2010. The Discharge of Fine Silica Sands in a Silo. Physics of Fluids 22, 043306.

[Klinzing et al. 2010] Klinzing, G.E., F. Rizk, R. Marcus, and L. S. Leung. 2010. "Pneumatic Conveying of Solids A Theoretical and Practical Approach," Third Edition, Springer, New York; and references therein.

[Li et al. 2009] Li, H.Z., J. Wang, and J. M. Fan. 2009. Analysis and Modeling of Particle Velocities in Micro-Abrasive Air Jet. International Journal of Machine Tools and Manufacture 49, 850-858; and references therein.

[Musgrove et al. 2009] Musgrove, G.O., M. D. Barringer, K. A. Thole, E. Grover, and J. Barker. 2009. Computational Design of a Louver Particle Separator for Gas Turbine Engines. Proceedings of ASME Turbo Expo 2009, Paper No. GT2009-60199.

[Parslow et al. 1999] Parslow, G.I., D. J. Stephenson, J. E. Strutt, and S. Tetlow. 1999. Investigation of Solid Particle Erosion in Components of Complex Geometry. Wear 233-235, 737-745.

[Sæther et al. 2009] Sæther, A., C. Arakaki, C. Ratnayake and D. Di Ruscio. 2009. Prediction of Mass Flow Rate in Pneumatic Conveying Using a System Identification Modeling Approach. Particulate Science and Technology 27, 314-326. 
[Tabakoff 1989] Tabakoff, W. 1989. Investigation of Coatings at High Temperature for Use in Turbomachinery. Surface and Coatings Technology 39-40, 97-115.

[Wang and Li 2003] Wang L., and D. Y. Li. 2003. Mechanical, Electrochemical and Tribological Properties of Nanocrystalline Surface of Brass Produced by Sandblasting and Annealing. Surface and Coatings Technology $167,188-196$.

[Wright 1987] Wright, I.G., 1987. High Temperature Erosion in Coal Combustion and Conversion Processes: Review. Materials Science and Engineering 88, 261-271.

[Wright et al. 2000] Wright, I.G., C. Leyens, and B. A. Pint. 2000. An Analysis of the Potential for Deposition, Erosion or Corrosion in Gas Turbines Fueled by the Products of Biomass Gasification or Combustion. Proceedings of the ASME Turbo Expo 2000, Paper No. 2000-GT-0019.

[Wypych 2008] Wypych, P. W. 2008. Dense-Phase Pneumatic Conveying Technology for Highly Explosive Metal Powders. Particulate Science and Technology 26, 15-22.

[Yan and Koplik 2009] Yan, Y., and J. Koplik. 2009. Transport and Sedimentation of Suspended Particles in Inertial Pressure-Driven Flow. Physics of Fluids 21, 013301. 
Figure 1: Conceptual illustration of the system studied. The dynamometer can be replaced (e.g., by an electric generator, mechanical transmission, or hydraulic pump), depending on the form of output energy desired. A volume $V$ of two-phase fluid increases as it accelerates down the nozzle.

Figure 2: Photograph of some of the primary components of the experimental apparatus. Shown from the top are a bottom fitting of the discharge tank, a valve, the nozzle, the top plate of the particle separation chamber, and the turbine. On the right is an exhaust hose.

Table 1: Sample Parameter Values. Gas: nitrogen. Nozzle: 304 stainless steel. Solid particles: cast stainless steel. Turbine: 316 stainless steel.

$\underline{\text { Parameter }}$

$r_{g}$ (gas molecule effective radius)

$r_{s}$ (solid particle approximate radius)

$r_{n}$ (nozzle radius)

$L_{n}$ (nozzle length)

$T$ (temperature during isothermal expansion)

$m_{g}$ (gas molecule mass)

$\rho_{m s}$ (solid material mass density)

$R_{\kappa}=\kappa_{s} / \kappa_{g}$ (solid to gas drift kinetic energy ratio)

$\eta$ (dissipative loss factor)

$v_{s f}$ (maximum solid-particle speed)

$P_{f}$ (maximum fluid kinetic power)

$c_{g}$ (gas specific heat capacity)

$c_{s}$ (solid specific heat capacity)

$R_{C}=C_{s} / C_{g}$ (solid to gas ratio of heat capacities)

$\gamma$ (ratio of gas heat capacities)

$K_{g}$ (gas thermal conductivity)

$K_{s}$ (solid thermal conductivity)

$f_{\Delta T f}=\Delta T_{f} / T$ (fractional change of gas temperature)

$\underline{\text { Parameter }}$

$p$ (fluid pressure)

$R_{V}=V_{s} / V_{g}$ (solid to gas volume ratio)

$n_{g}^{-1 / 3}$ (typical distance nearest-neighbor gas molecules)

$n_{s}^{-1 / 3}$ (typical distance nearest-neighbor solid particles)

$\lambda_{g}$ (molecule-molecule collision mean free path)

$\lambda_{s}=\lambda_{g s} / 4$ (particle-particle collision mean free path)

$\lambda_{g s}$ (molecule-particle collision mean free path)
Value Within Nozzle

$0.16 \mathrm{~nm}$

$100 \mu \mathrm{m}$

$5 \mathrm{~mm}$

$30 \mathrm{~cm}$

$298 \mathrm{~K}$

$28 \mathrm{u}$

$8000 \mathrm{~kg} / \mathrm{m}^{3}$

1200

1

$17 \mathrm{~m} / \mathrm{s}$

$240 \mathrm{~W}$

$743 \mathrm{~J} / \mathrm{kg} \cdot \mathrm{K}$

$500 \mathrm{~J} / \mathrm{kg} \cdot \mathrm{K}$

810

1.4

$0.025 \mathrm{~W} / \mathrm{m} \cdot \mathrm{K}$

$16 \mathrm{~W} / \mathrm{m} \cdot \mathrm{K}$

$\sim 10^{-2}$

Initial Value

$0.79 \mathrm{MPa}$

1.35

$2.3 \mathrm{~nm}$

$190 \mu \mathrm{m}$

$38 \mathrm{~nm}$

$58 \mu \mathrm{m}$

$230 \mu \mathrm{m}$ $\underline{\text { Final Value }}$ $0.10 \mathrm{MPa}$ 0.17

$3.6 \mathrm{~nm}$

$310 \mu \mathrm{m}$

$150 \mathrm{~nm}$

$230 \mu \mathrm{m}$

$920 \mu \mathrm{m}$ 


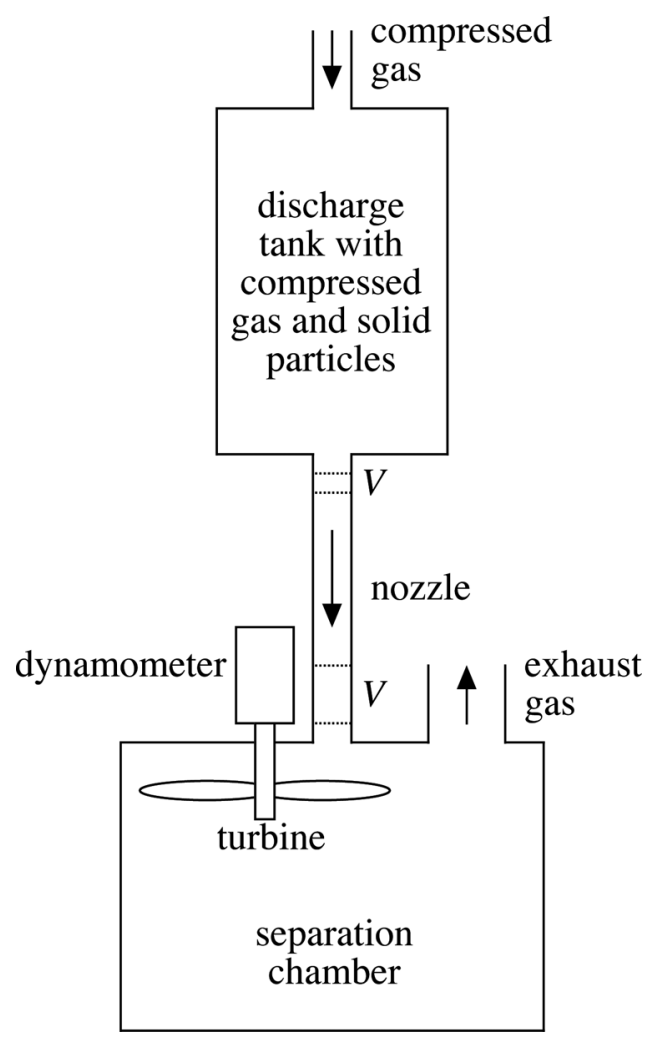




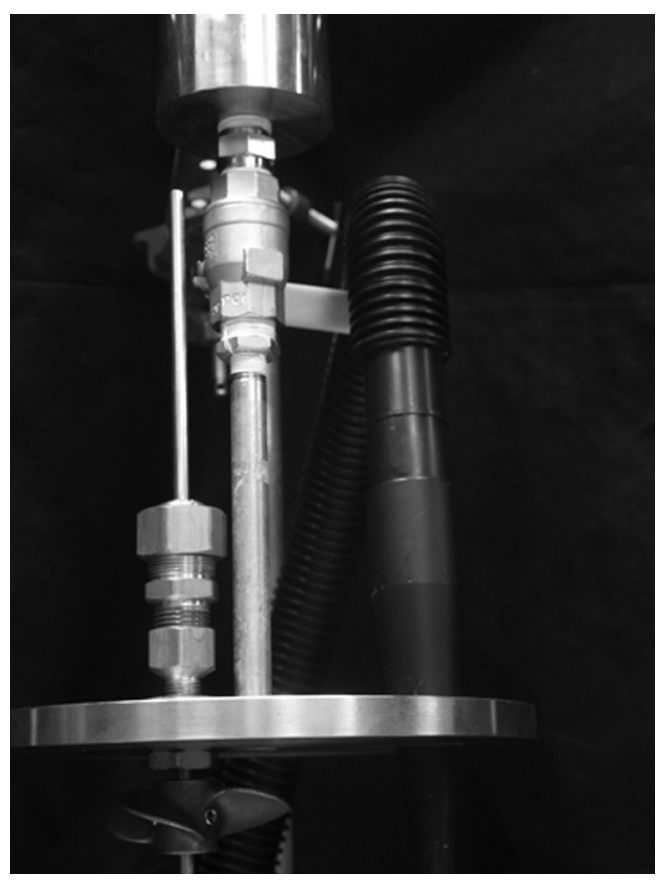

